\title{
Landschaftsschäden im Zusammenhang mit dem Skisport
}

Jedermann weiß, welch enormen Aufschwung der Skisport in der jüngeren Vergangenheit erlebt hat und welche Bedeutung dieser Freizeitbeschäftigung heute für die Gesundheit des einzelnen Menschen sowie für die Wirtschaft als Ganzes zukommt. Weniger bekannt ist dagegen die Tatsache, daß im Zusammenhang mit dem Skisport Landschaftsschäden entstehen, die zum Teil recht erheblich sind. Dies ist eines der zahlreichen Ergebnisse einer Diplomarbeit unter dem Titel «Landschaftsschäden und Entschädigungspraxis im Zusammenhang mit dem Skisport», die im Frühjahr/Sommer 1976 von einem Absolventen des Schweizerischen Landwirtschaftlichen Technikums in Zollikofen erstellt wurde. Deren Autor, Alex Pfiffner (aus Weißtannen), füllt mit seinem 165 seitigen Werk eine echte Marktlücke, liegen doch zu diesem Thema in der Schweiz noch keine umfassenden Untersuchungen vor. Es ist deshalb zu hoffen, daß diese Arbeit gerade auch seitens der Geographie und des Landschaftsschutzes - Beachtung finden und $A$ la $B$ zu weiteren Forschungen und Überlegungen geben wird.

\section{Einfluß der Pistenpräparierung und Pisten- benutzung auf den Ernteertrag}

Durch die Pistenpräparierung mit Pistenfahrzeugen wird der Schnee zusammengedrückt. Dieser Verdichtung der Schneedecke hilft der Skifahrer durch das ständige Benützen der Skipiste noch zusätzlich nach. Durch diesen Druck entsteht eine Eisdecke, die je nach Witterungsverhältnissen und Pistenfrequentierung verschieden dick sein kann. Regnet es im Verlauf des Winters auf die Piste und folgt nachher eine Kälteperiode, wird die Eisbildung besonders gefördert. Eine entscheidende Rolle spielen dabei auch die Höhe der Schneedecke und die Exposition des Geländes. Der größte Prozentsatz der Ertragsausfälle für die Berglandwirtschaft geht auf das Konto der Pistenpräparierung. Unter der verfestigten Piste gehen viele wertvolle Futterpflanzen zugrunde. Dies ist nicht nur eine Folge der Verdichtung des Bodens, sondern auch des Sauerstoffmangels unter der Schneedecke, denn dadurch treten Erstickungs- und Fäulniserscheinungen auf. Zudem bewirkt die Eisdecke, daß die Skipistenflächen im Frühjahr später ausapern, was einen Vegetationsrückstand zur Folge hat. Dies wirkt sich negativ auf den Ernteertrag aus. Die Zeit zur Erholung

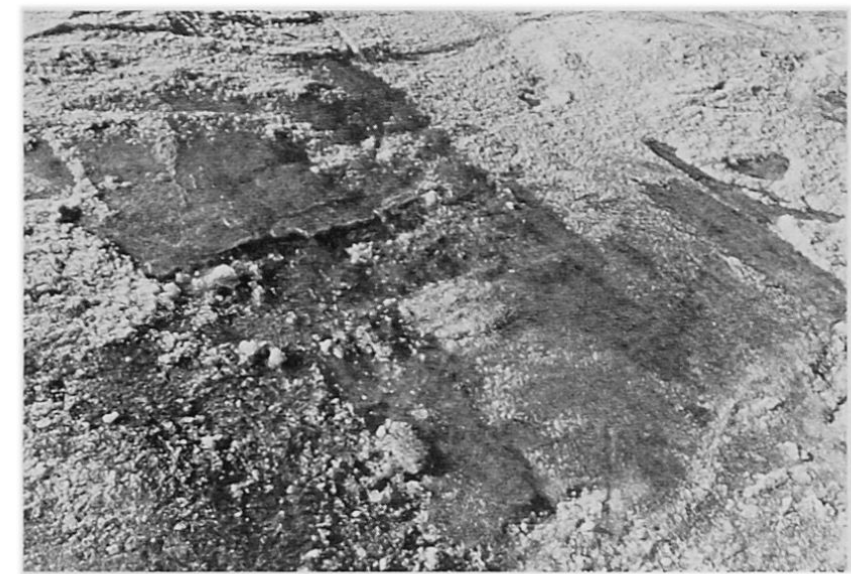

Eisbildung auf einer Abfahrtspiste im Pizolgebiet. Ortsbestimmung: Gondelbahn Wangi-Pizol (Furt) Aufnahme: 3. 3.1976

während der kurzen Vegetationsperiode ist vor allem in höheren Lagen in der Regel zu kurz, so daß es unter vielbefahrenen Pisten bald einmal zu einem dauernden Ertragsausfall kommt.

Die mechanischen Schäden, welche die Pistenfahrzeuge anrichten, sind im allgemeinen relativ klein. Wenn jedoch bei zu geringer Schneedecke und in sehr steilen Hängen gefahren wird, können die Schäden erheblich sein.

\section{Verletzung der Grasnarbe durch die Skikanten}

Solche Schäden treten normalerweise nur bei geringer Schneehöhe, bei Talstationseinläufen und in Engpässen auf. Aber auch bei hohem Schnee können junge Waldpflanzen und Sträucher, die zum Zweck der Lawinensicherung oder Walderneuerung gepflanzt wurden oder natürlich aufgekommen sind, durch die scharfen Skikanten verletzt werden.

In der Regel sind diese Schäden im flachen Gelände gering, in Steilhängen und im kupierten Gelände jedoch größer. Skifahrer, denen gewisse Hänge zu steil sind, rutschen oft quer auf den Kanten den Hang hinunter. Dadurch wird der Schnee häufig bis auf die Grasnarbe abgeschabt. Da der Boden gefroren ist, kann weiterhin auf der Grasnarbe abgerutscht werden,

Dr. Hans Moor, Schweiz. Landw. Technikum, Länggasse 85, 3052 Zollikofen 


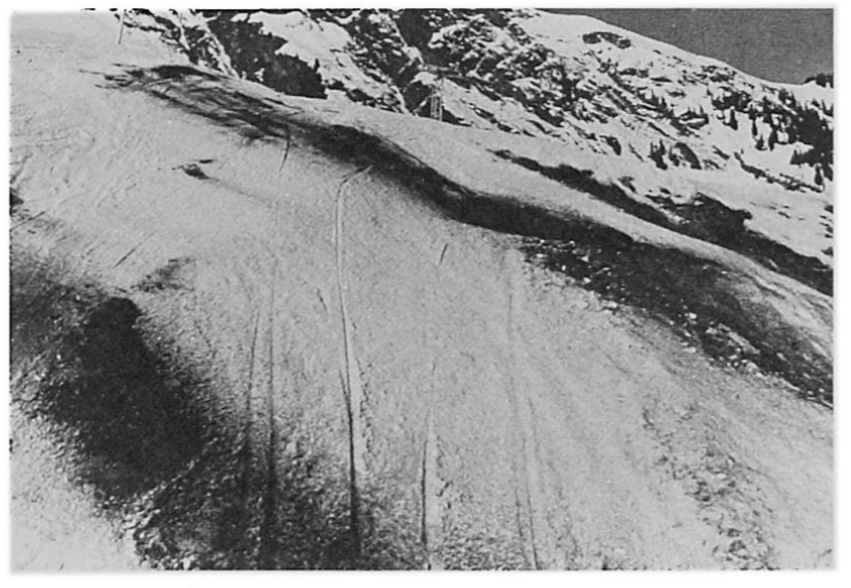

L.andschaftsschäden infolge Schneemangels.

Ortsbestimmung: Pizolgebiet beim Skilift Furt-Gaffia

Aufnahme: 3. 3. 1976

wodurch diese erheblich verletzt oder sogar vollständig abrasiert wird. Zudem kommt es relativ häufig vor, daß in Kurven, an erhöhten Stellen und Wegbordrändern der Schnee bis auf den Boden weggeschabt wird, so daß an solchen Orten oft ein 100\%iger Ertragsausfall hingenommen werden muß.

Ferner können große Schäden entstehen, wenn im Frühjahr bei wenig Schnee noch Ski gefahren wird. Oft kommt es dann vor, daß die höher gelegenen Anlagen für den Skibetrieb noch genügend Schnee aufweisen, die Pisten der unteren Sektionen jedoch stellenweise bereits schneefrei sind, trotzdem aber befahren werden.

\section{Liegengelassene Gegenstände und Abfall}

Mancher Skifahrer weiß aus Erfahrung, daß man verlorene Gegenstände im Schnee häufig nicht leicht wieder findet. Sofern diese Gegenstände im Frühjahr nicht restlos eingesammelt werden, können sie in die Mähmaschine gelangen und diese beschädigen. Glasscherben von Skibrillen und weggeworfenen Flaschen bilden nicht nur eine ernste Verletzungsgefahr für Mensch und Tier, sondern bringen unter Umständen auch Pneus von Erntemaschinen zum Platzen, was in Steilhängen eine erhebliche Unfallgefahr bedeutet. In der Nähe von Bergrestaurants, bei Ruhebänken und am Rande von Skipisten wird oft eine große Menge Abfall liegengelassen, so daß im Frühjahr die
Wiesen in Unordnung angetroffen werden. Papierabfälle verschmutzen das Futter. Konservenbüchsen und Flaschensplitter bedeuten eine große Verletzungsgefahr für das Vieh, ebenso wie die Fremdkörper, welche durch das Futter vom Tier aufgenommen werden.

\section{Erdplanierungen und Waldrodungen zum Zweck der Pistenverbesserung}

In den letzten Jahren ist es üblich geworden, in Berggebieten, die durch Sportbahnen erschlossen werden, Skipistenplanierungen vorzunehmen. Ein wichtiges Motiv solcher Planierungen besteht darin, gefährliche Stellen zu sanieren und vorstehende Steine wegzuräumen, damit die Skipiste auch bei geringer Schneemenge befahren werden kann. Dadurch wird die Leistungsfähigkeit und Rendite der Anlagen wesentlich erhöht.

Auf breit ausplanierten Pisten kann sich auch der Anfänger herumtummeln, ohne andere zu behindern. Zweifellos wird dadurch die Unfallgefahr gesenkt. Anderseits werden aber wieder neue Gefahren - wie zu schnelles Fahren - heraufbeschworen.

Die Unsitte, unberührtes Erholungsgelände zu Skibahnen umzufunktionieren, griff anfangs der siebziger Jahre auf die Schweiz über. Heute trifft man in verschiedenen Skigebieten zum Teil unrühmliche Schutthalden an. Sie mögen im Winter als stark frequentierte Skibahnen ihre Berechtigung haben - im Sommer und Herbst bedeuten sie mancherorts eine Verschandelung schönsten Erholungsraumes.

Häufig ist man sich zu wenig bewußt, was es braucht, bis die zerstörte Vegetation rekultiviert ist. Unter den schwierigen Bedingungen oberhalb der Waldgrenze faßt eine widerstandsfähige Vegetation oft nur schwer Fuß. Dort, wo der Humus wegplaniert wurde, kann es - nach Ansicht von Fachleuten - 20 und mehr Jahre dauern, bis die «Wunden» geheilt sind.

Die alpine Vegetation sichert die steilen Hänge vor Erosion und Auswaschung. Ist diese einmal zerstört, kann der Humus sehr schnell ausgeschwemmt und in die Tiefe getragen werden. Weil das Wasser nicht mehr durch die Vegetation zurückgehalten wird, fließt es schneller ab. Dies kann bewirken, daß tiefe Erosionsgräben entstehen und ganze Hänge ins Rutschen geraten. Nicht nur bei Pistenplanierungen können 


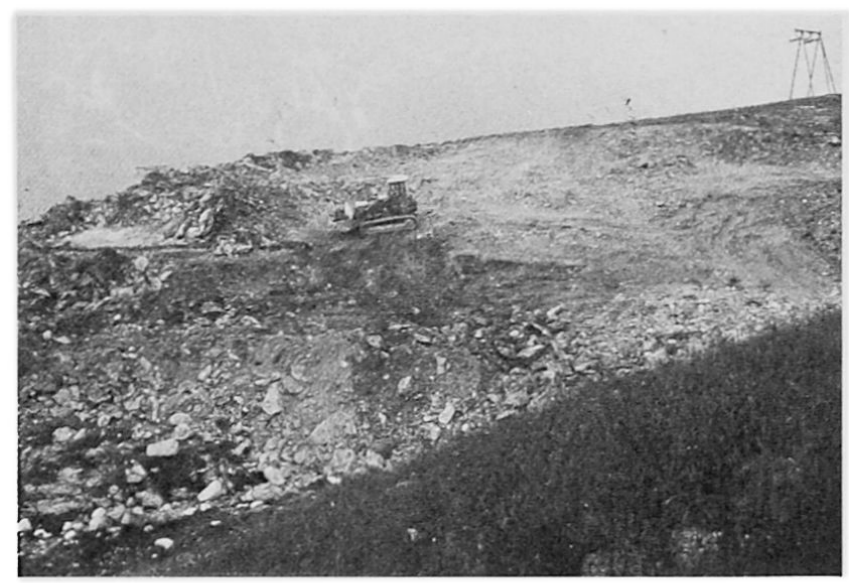

Landschaftsschäden durch Erdplanierungen.

Ortsbestimmung: Piz Nair-Gebiet St. Moritz, Alp Giop

Aufnahme: 21. 7.1976

Erosionsherde entstehen, sondern auch beim Erstellen von Anlagen, wie zum Beispiel einzelner Stationen, Mastenfundamente, Leitungs- und Kabelgräben. Aber auch die Lagerung der oft beträchtlichen Aushubmassen in Steilhängen und ihr geländegerechter Dauereinbau können Schwierigkeiten bereiten.

Bei der Erstellung einer Skipiste, beim Bau von Skiliften und Seilbahnen kommt es häufig vor, daß Wald gerodet werden muß. Obwohl nach dem Eidgenössischen Forstgesetz die gerodete Waldfläche anderweitig wieder angepflanzt werden muß, kann die Veränderung eines Waldrandes für die übrige Waldfläche durchaus negative Folgen zeitigen - zum Beispiel eine wesentliche Erhöhung des Windwurfrisikos.

\section{Ausmaß der Ertragsausfälle durch den Skipistenbetrieb}

1972 wurden in der Gegend von Davos erstmals exakte Ertragserhebungen durchgeführt, aufgrund derer in einem konkreten Einzelbeispiel Ertragsausfälle von nicht weniger als $64 \%$ festgestellt wurden. Solche Einbußen sind ohne Zweifel Extremfälle. Zudem unterliegen sie von Gebiet zu Gebiet und von Jahr zu Jahr starken Schwankungen. Aufgrund eigener Erhebungen kommt Alex Pfiffner in seiner Diplomarbeit zum Schluß, daß die Ertragsausfälle im Durchschnitt der letzten Jahre schätzungsweise 15 bis $25 \%$ betragen haben.

\section{Botanische Veränderungen als Folge des Pistenbetriebs}

Die Ursache von botanischen Veränderungen liegt in der Pistenpräparierung, welche die Bildung einer Eisschicht bewirkt. Durch die Bodenverdichtung und den Sauerstoffmangel werden vor allem die feinen Untergräserarten geschädigt und weichen den standfesteren Kräutern. Auch werden wertvolle Kräuter durch minderwertige Arten verdrängt. Der auf Bergwiesen üblicherweise geringe Anteil an Klee wird durch den Pistenbetrieb noch kleiner.

Entscheidend ist aber die Zunahme der Kräuter und die Abnahme der Gräser, wodurch der Futterwert verschlechtert wird. Kräuterreiches Futter braucht zudem mehr Zeit zur Trocknung; daher können große Nährstoffverluste eintreten. Außerdem sind die Brökkelverluste bei kräuterreichem Futter größer. Schließlich bedingt eine längere Bodentrocknung oft einen größeren Arbeitsaufwand. Bei der Benützung einer Heubelüftungsanlage steigen die Stromkosten infolge der längeren Belüftungszeit.

Obwohl es schwierig ist, die Qualitätsverschlechterung wertmäßig exakt zu beziffern, sollte bei der Entschädigung von Ertragseinbußen, neben dem quantitativen Ausfall, auch die Verschlechterung der Futterqualität berücksichtigt werden.

Futterverschlechterung als Folge des Pistenbetriebs (Durchschnittsergebnisse aus Versuchen in Wasserwendi, Gemeinde Hasliberg, sowie auf der Skipiste der Luftseilbahn Reuti-Mägisalp im Hasliberg)

Zeit: 2. 6. 1976

Botanische Zusammensetzung neben der Skipiste:

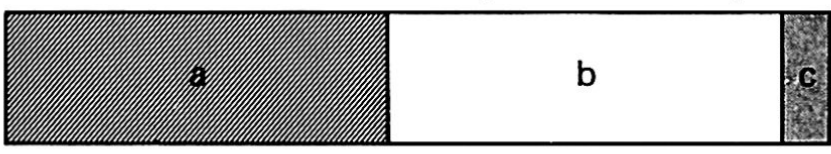

Botanische Zusammensetzung in der Skipiste:

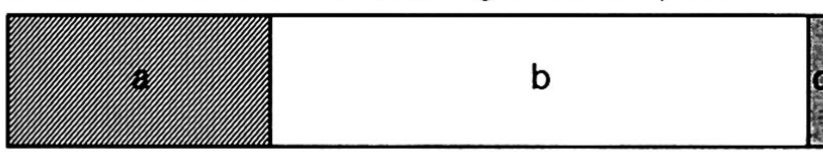
a = Gräseranteil:
$46,7 \%$
bzw. $31,4 \%$
b $=$ Kräuteranteil:
$47,4 \%$
bzw. $66,3 \%$
$c=$ Kleeanteil:
$\frac{5,9 \%}{100,0 \%}$ bzw. $\frac{2,3 \%}{100,0 \%}$ 


\section{Die heutige Entschädigungspraxis}

Obschon nach Art. 699 des Zivilgesetzbuches jedermann das Recht zusteht, Wald und Weide zu betreten, sehen verschiedene kantonale und kommunale Erlasse eine Entschädigungspflicht für verursachten Schaden oder für die Auferlegung eines Bauverbots innerhalb der Skipiste vor. Diese Bestimmungen weichen jedoch von Kanton zu Kanton und von Gemeinde zu Gemeinde stark voneinander ab. Vielfach sind sie auch materiell ungenügend oder fehlen überhaupt. Eine einheitliche(re) Regelung auf kantonaler oder eidgenössischer Ebene drängt sich auf, gaben doch bei der von Alex Pfiffner durchgeführten Direktbefragung $64 \%$ der Skipisten-Grundeigentümer an, überhaupt keine Entschädigungen zu erhalten.

Da gerade im Kanton Graubünden vielerorts keinerlei Entschädigungen entrichtet wurden, kam es 1973 zur Gründung der «Vereinigung der Skipisten-Grundeigentümen». Dieser Organisation ist es 1974 gelungen, mit der «Vereinigung der Seilbahn- und Skiliftunternehmungen» eine Vereinbarung abzuschließen betreffend das Verfahren für die Feststellung von Schäden an landwirtschaftlichen Grundstücken. Diese Übereinkunft stellt ohne Zweifel einen Fortschritt dar; es haften ihr jedoch noch verschiedene Mängel an.

\section{Dienstbarkeitsverträge und Entschädigungsmodus}

Der Dienstbarkeitsvertrag zwischen dem Sportbahnunternehmen und dem einzelnen Skipisten-Grundeigentümer bildet die Basis für die Entschädigungsleistungen. In diesem Vertrag müssen alle Einzelheiten genau geregelt werden. Zudem sollte durch Aufnahme einer entsprechenden Bestimmung im Vertragstext die Möglichkeit eingeräumt werden, bei Einwilligung beider Parteien in einem späteren Zeitpunkt Zusatzregelungen zum bestehenden Vertrag anzubringen.

Um den Aufwand bei der Schadenerhebung möglichst klein zu halten, sollten für den Ertragsausfall Durchschnittswerte ermittelt werden, die dann für mehrere Jahre die Basis der Entschädigung bilden. Die Vergütungen für Masten und alle weiteren Benachteiligungen müssen nach einheitlichen Richtlinien festgelegt werden. Mit Ausnahme der einmaligen Masten- entschädigung sind alle sonstigen Vergütungen vorteilhaft jährlich auszurichten.

In größeren Wintersportzentren muß verlangt werden, daß die Entschädigungen für Pistendurchleitungen und Ertragsausfälle nicht nur durch die Sportbahnunternehmungen, sondern auch von den übrigen interessierten Kreisen - Hotellerie, Kur- und Verkehrsverein, Skischule und Gemeinde - zu übernehmen sind.

\section{Schlußbemerkungen}

Im Zusammenhang mit dem Skisport entstehen für die Berglandwirtschaft Landschaftsschäden und Behinderungen in der Bewirtschaftung, die zum Teil recht erheblich sind. Die heutige Entschädigungspraxis ist von Kanton zu Kanton und von Gemeinde zu Gemeinde sehr unterschiedlich; vielfach ist sie auch materiell ungenügend. Es wäre wünschbar, wenn die zahlreichen in der Diplomarbeit von Alex Pfiffner unterbreiteten, aus Platzgründen hier lediglich angedeuteten Forderungen und Vorschläge ernsthaft geprüft und zu einer angemessenen und einheitlicheren Entschädigung der betroffenen Skipisten-Grundeigentümer führen würden.

Im Rahmen der vorliegenden Arbeit zum Thema «Landschaftsschäden und Entschädigungspraxis im Zusammenhang mit dem Skisport» zeigt sich erneut, wie komplex die von der Berglandwirtschaft ausgeübten Funktionen im Grunde genommen sind. Die Landwirtschaft produziert eben nicht nur Nahrungsmittel. Zusätzlich erfüllt sie wichtige Nebenfunktionen, deren Bedeutung in einer hochentwickelten Volkswirtschaft, wie der unsrigen, ständig zunimmt. 